\title{
ERRATUM: Comparison between Craniospinal Irradiation and Limited-Field Radiation in Patients with Non-metastatic Bifocal Germinoma
}

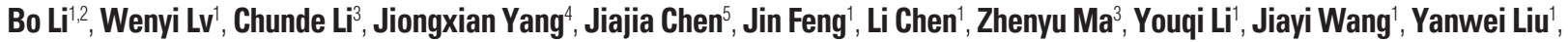 \\ Yanong Li ${ }^{1}$, Shuai Liu', Shiqi Luo ${ }^{3}$, Xiaoguang Qiü,2 \\ ${ }^{1}$ Department of Radiation Oncology, Beijing Tiantan Hospital, Capital Medical University, Beijing, ${ }^{2}$ Beijing Neurosurgical Institute, Capital Medical \\ University, Beijing, ${ }^{3}$ Department of Neurosurgery, Beijing Tiantan Hospital, Capital Medical University, Beijing, ${ }^{4}$ Department of Clinical Nutrition, \\ Beijing Children's Hospital, Capital Medical University, Beijing, ${ }^{5}$ Centre of Endocrinology Genetics and Metabolism, National Centre for Children's \\ Health, Beijing Children's Hospital, Capital Medical University, Beijing, China
}

At the end of second paragraph of "Materials and Methods" section, the original sentence is "The clinical target volume (CTV) was obtained by adding $0.5 \mathrm{~cm}$ to GTV. Additional $0.5-1 \mathrm{~cm}$ was added to CTV to create planning target volume (PTV)." The correct one should be "The clinical target volume (CTV) was obtained by adding 0.5-1 cm to GTV. Additional $0.3-0.5 \mathrm{~cm}$ was added to CTV to create planning target volume (PTV)." The change does not affect the outcome of the original paper. On behalf of all the co-authors in this paper, I deeply apologize for my lack of oversight and missing this error.

*DOI of original article: http:// dx.doi.org/10.4143/crt.2020.437 